\title{
Continuously Tunable Emission in Inverted Type-I CdS/ CdSe Core/Crown Semiconductor Nanoplatelets
}

\author{
Savas Delikanli, Burak Guzelturk, Pedro L. Hernández-Martínez, Talha Erdem, \\ Yusuf Kelestemur, Murat Olutas, Mehmet Zafer Akgul, and Hilmi V. Demir*
}

The synthesis and unique tunable optical properties of core/crown nanoplatelets having an inverted Type-I heterostructure are presented. Here, colloidal 2D $\mathrm{CdS} / \mathrm{CdSe}$ heteronanoplatelets are grown with thickness of four monolayers using seed-mediated method. In this work, it is shown that the emission peak of the resulting $\mathrm{CdS} / \mathrm{CdSe}$ heteronanoplatelets can be continuously spectrally tuned between the peak emission wavelengths of the core only CdS nanoplatelets (421 nm) and CdSe nanoplatelets (515 nm) having the same vertical thickness. In these inverted Type-I nanoplatelets, the unique continuous tunable emission is enabled by adjusting the lateral width of the $\mathrm{CdSe}$ crown, having a narrower bandgap, around the core CdS nanoplatelet, having a wider bandgap, as a result of the controlled lateral quantum confinement in the crown region additional to the pure vertical confinement. As a proof-of-concept demonstration, a white light generation is shown by using color conversion with these $\mathrm{CdS} / \mathrm{CdSe}$ heteronanoplatelets having finely tuned thin crowns, resulting in a color rendering index of 80 . The robust control of the electronic structure in such inverted Type-I heteronanoplatelets achieved by tailoring the lateral extent of the crown coating around the core template presents a new enabling pathway for bandgap engineering in solution-processed quantum wells.

understanding fundamental excitonic processes but also because of their potential important applications in light-emitting diodes (LEDs), ${ }^{[1]}$ lasers, ${ }^{[2]}$ photovoltaics, ${ }^{[3]}$ biological imaging, ${ }^{[4]}$ and spintronics. ${ }^{[5]}$ The rapidly developing field of colloidal synthesis now makes custom designs of nanocrystals with a precise control over the size, shape, and composition possible. To date, semiconductor nanocrystals of various shapes including spherical dots, ${ }^{[6]}$ nanorods, ${ }^{[7]}$ tetrapods, ${ }^{[8]}$ nanowires, ${ }^{[9]}$ nanoribbons, ${ }^{[10]}$ and most recently nanoplatelets (NPLs) ${ }^{[11]}$ have been successfully synthesized. In these solution-processed quantum structures, an additional epitaxial growth of semiconductor shell around the starting semiconductor core leads to various architectures of nanocrystal heterostructures. By doing so, physical properties can be elegantly modified with precisely controlling distribution of the composition across the heterostructure. These colloidal heteronanocrystals

\section{Introduction}

Colloidal semiconductor nanocrystals have become increasingly more prominent not only because of their importance in

Dr. S. Delikanli, B. Guzelturk,

Dr. P. L. Hernández-Martínez, T. Erdem,

Y. Kelestemur, Dr. M. Olutas, M. Z. Akgul,

Prof. H. V. Demir

Department of Electrical and Electronics Engineering

Department of Physics

UNAM - Institute of Materials Science and

Nanotechnology

Bilkent University

Ankara 06800, Turkey

E-mail: volkan@bilkent.edu.tr; hvdemir@ntu.edu.sg

B. Guzelturk, Dr. P. L. Hernández-Martínez, Prof. H. V. Demir

LUMINOUS! Center of Excellence for Semiconductor Lighting and

Displays

School of Electrical and Electronic Engineering

School of Physical and Materials Sciences

Nanyang Technological University

Singapore 639798, Singapore

Dr. M. Olutas

Department of Physics

Abant Izzet Baysal University

Bolu 14280, Turkey

DOI: 10.1002/adfm.201500403 are possibly the best candidates for excitonic engineering and present attractive opportunities for enhanced platforms of colloidal photonics. ${ }^{[12]}$ Previously, various solution-processed core/ shell quantum dots and rods have been studied for excitonically engineered properties. For example, Type-I CdSe/ZnSe core/ shell nanocrystals ${ }^{[13]}$ and $\mathrm{ZnSe} / \mathrm{CdSe}$ core/shell nanocrystals tunable between inverted Type-I and Type-II ${ }^{[14]}$ were reported. With precise control of the shell, optical properties of core-only nanocrystals including quantum yield, ${ }^{[15]}$ photostability, ${ }^{[16]}$ and reduction of fluorescence emission blinking ${ }^{[17,18]}$ can be greatly enhanced, which thus make them highly attractive for colloidal LEDs, ${ }^{[19]}$ colloidal lasers, ${ }^{[20]}$ and biological imaging. ${ }^{[17]}$

Recently, novel inverted Type-I nanocrystal heterostructures have drawn considerable interest thanks to their high charge injection efficiency and enhanced absorption range which can be exploited in optoelectronic devices especially for applications in photovoltaics and photodetection. ${ }^{[21,22]} \mathrm{CdS} / \mathrm{HgS}^{[23]}$ and $\mathrm{CdS} /$ $\mathrm{CdSe}^{[21,24]}$ spherical core/shell architectures have been the most studied inverted Type-I colloidal nanoparticles, in each of which a thin layer of narrower bandgap material was grown onto a wider bandgap colloidal quantum dot forming a quantum dot-quantum shell heterostructure. In these architectures, quantum confinement in the quantum shell arises from only one direction, radial, with the condition that the perimeter of the shell has to be considerably larger than the radius of the 
exciton of the narrower bandgap shell material. The synthesis of $\mathrm{CdS} / \mathrm{HgS}$ core/shell is found to be problematic mainly due to the undeveloped $\mathrm{HgS}$ shell growth techniques and their optical properties are poor as well. Although CdS/CdSe system offers relatively uniform size distribution, their quantum yield still remains low, especially with the thin layers of CdSe shell.

With their robust control of the shell and crown growth on the seed NPLs, ${ }^{[25-27]}$ tunable thickness at the monolayer (ML) level, ${ }^{[11,28]}$ and exceptional optical features including narrow emission on the order of $35 \mathrm{meV}^{[28]}$ and quantum yield as high as $80 \%$ at room temperature, ${ }^{[29]}$ NPLs stand out as a unique ground for studies of various heterostructures. Although Type-I core/crown heterostructures of NPLs emitting spectrally at the same position as only core NPLs have been reported by several groups, ${ }^{[25,27,29,30]}$ to the best of our knowledge, there has been no report of 2D heteronanoplatelets with inverted Type-I electronic structure having tunable band edge emission to date.

In this paper, we report the synthesis and characterizations of the first examples of CdS/CdSe core/crown NPLs having inverted Type-I heterostructure. Atomically flat CdSe crown having the exact same number of atomic layers in the vertical direction was grown laterally using core-seeded method. We studied the excitonic and optical properties of these inverted Type-I NPLs by systematically varying the lateral size of the narrow bandgap semiconductor, CdSe crown. We showed that photoluminescence (PL) can be tuned continuously between the emission wavelengths of the core only CdS and CdSe NPLs having the same vertical thickness by only controlling the lateral extent of the crown. Compared to the core only CdS NPLs, the resulting CdS/CdSe NPLs exhibit superior quantum yield as high as $55 \%\left(\lambda_{\text {exc }}=400 \mathrm{~nm}\right)$ estimated by following the method developed by de Mello et al. ${ }^{[31]}$ owing to the efficient internal exciton funneling from the $\mathrm{CdS}$ core into the CdSe crown and higher quantum efficiency of the CdSe crown. Also, preparing conventional Type-I CdSe/CdS core/crown NPLs, we compared optical properties to develop a better understanding of the excitonic processes in inverted Type-I CdS/CdSe NPLs. Furthermore, we performed time-resolved fluorescence (TRF) measurements at the band edge emission peak to understand the decay kinetics of the emission. Finally, we investigated the potential use of these NPLs with thin crowns in white light generation. We showed that the generated white light promises a reasonable color rendering performance along with exhibiting a comparable color correlated temperature (CCT) to the natural sunlight.

\section{Results and Discussion}

We used seed-mediated growth to synthesize the CdS/CdSe core/crown NPLs. Here, first as the seed, 4 ML CdS NPLs were grown with slight modifications from the synthesis in the literature. ${ }^{[32]}$ Then, subsequent growth of CdSe crown was achieved by continuous injection of selenium precursor, elemental Se well dissolved in octadecene (ODE), and cadmium precursor, cadmium acetate homogenously dispersed in a mixture of oleic acid (OA) and ODE. The injection amount was tuned to control lateral size of the crown coating. Injection parameters including concentration of the injection solution, injection speed, temperature, and concentration of the seeds needed to be carefully adjusted. For example, higher injection rates led to separate nucleation of CdSe NPLs and low injection temperatures prevented the lateral growth. Further details of the synthesis are given in the Experimental Section.

Length of the starting CdS NPL seeds along the direction they rolled up was measured to be $45 \pm 6 \mathrm{~nm}$ using high-angle annular dark-field transmission electron microscopy (HAADFTEM) images. An exemplary HAADF-TEM image of the CdS NPLs and an unfolded CdS NPL achieved by deposition of one monolayer CdS shell on each side of the NPL ${ }^{[27,33]}$ are shown in Figure 1a and the inset, respectively. PL and absorption spectra of these seed 4 ML CdS NPLs are presented in Figure 1b. The absorption maximum around $410 \mathrm{~nm}$ contains two peaks close to each other, the light hole transition and the heavy hole transition. ${ }^{[11]}$ Quantum yield of these CdS NPLs is very low, a few percent, compared to CdSe NPLs. The narrow PL peak at $421 \mathrm{~nm}(2.95 \mathrm{eV})$ with a full-width-at-half-maximum (FWHM) of $12 \mathrm{~nm}(83 \mathrm{meV})$ corresponds to the heavy hole transition while the broad emission located at $646 \mathrm{~nm}(1.92 \mathrm{eV})$ signifies the deep trap emission in these core only CdS NPLs. The FWHM of band edge emission of CdS NPLs is sharper than one can observe in colloidal spherical nanocrystals due to their pure $1 \mathrm{D}$ confinement while it is wider than that of the CdSe NPLs due to stronger temperature-dependent exciton-phonon interactions in the CdS NPLS. ${ }^{[29]}$ These seed CdS NPLs were precipitated and washed with addition of acetone, and dispersed in hexane before the lateral growth. For comparison, we also synthesized 4 ML CdSe NPLs with a slightly modified recipe from the literature. ${ }^{[1]}$ The resulting 4 ML CdSe NPLs exhibit sharp emission peak at $512 \mathrm{~nm}(2.42 \mathrm{eV})$, and heavy hole and light hole absorption peaks at $510 \mathrm{~nm}(2.43 \mathrm{eV})$ and $478 \mathrm{~nm}$ $(2.59 \mathrm{eV})$, respectively, as can be seen in Figure 1c.

In Type-I systems, although photons above the bandgap of the shell material are absorbed by both the core and shell regions, the emission is only observed from the narrower bandgap core semiconductor due to the rapid funneling of the excitons from the shell material with the wider bandgap into the core material with the narrower bandgap. In the CdSe/CdS core/shell heterostructures, holes are strongly localized in the CdSe region owing to the large valence band offset. ${ }^{[34]}$ However, the $\mathrm{CdSe} / \mathrm{CdS}$ core/shell heterostructures are widely recognized as quasi-Type-II because of strong electron delocalization due to the shallow conduction band offset. ${ }^{[34-36]}$ Absorption and PL spectra of the synthesized $4 \mathrm{ML} \mathrm{CdSe/CdS} \mathrm{core/crown} \mathrm{NPLS}$ are given in Figure 1d. Excitonic transitions of the $\mathrm{CdSe} / \mathrm{CdS}$ core/crown NPLs remain unchanged spectrally with the growth of CdS crown region compared to the core only CdSe and CdS NPLs due to the very small in-plane quantization.

The CdS/CdSe core/crown NPLs as mentioned earlier were synthesized by using the CdS NPLs as the seeds. We performed HAADF-TEM and X-Ray diffraction measurements on these CdS/CdSe NPLs to confirm the lateral growth of the CdSe crown on CdS NPLs. A HAADF-TEM image of CdS/ CdSe NPLs emitting at $515 \mathrm{~nm}$ is shown in Figure 2a. In the inset of Figure 2a, the HAADF-TEM image of unfolded CdS/ CdSe NPLs accomplished by one layer CdS shell deposition on each side of the NPLs is shown. The CdS/CdSe NPLs exhibit larger lateral length $(92 \pm 12 \mathrm{~nm})$ compared to the CdS core 
(a)

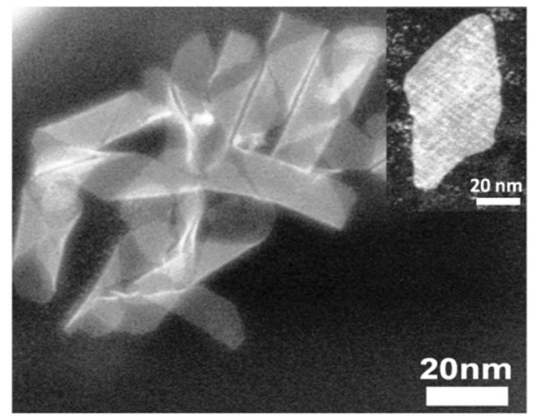

(c)

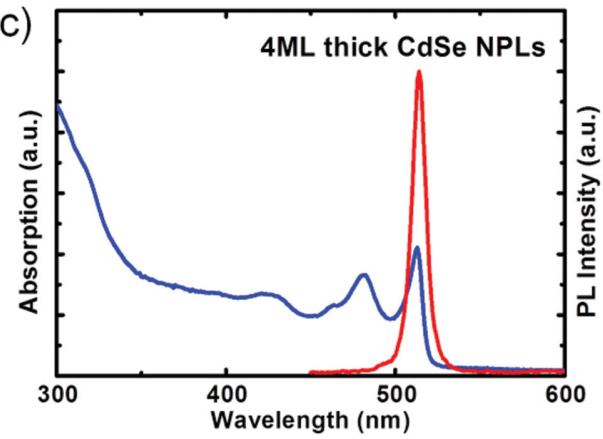

(b)

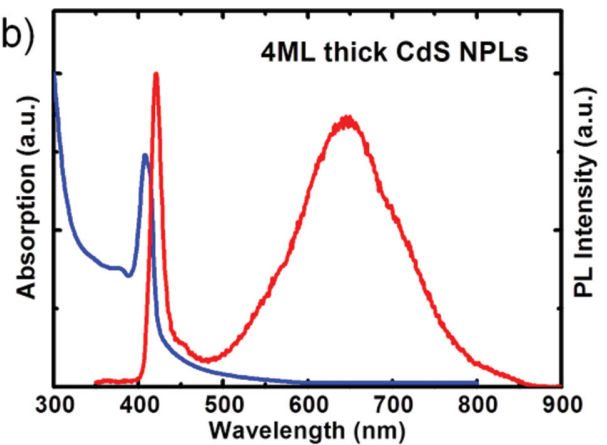

(d)

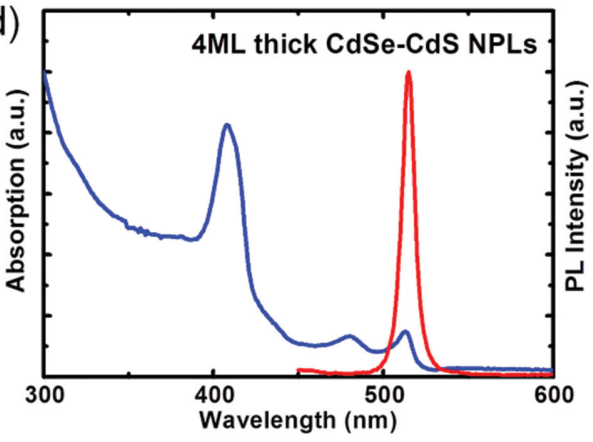

Figure 1. a) A HAADF-TEM image of 4 ML CdS NPLs. Absorption (blue) and PL (red) spectra of 4 ML thick b) CdS, c) CdSe, and d) CdSe/CdS NPLs.

only NPLs suggesting the lateral growth of CdSe crown on CdS NPLs. A HAADF-TEM image of a $4 \mathrm{ML}$ CdS/CdSe NPL coated with a thin layer of CdSe crown is given in Figure 2b. Yellow dashed line showing the boundary between $\mathrm{CdS}$ and $\mathrm{CdSe}$ layers was drawn to help the eyesight. Line EDX analysis given in Figure 2c shows the distribution of $\mathrm{S}$ and Se concentration along the blue line. As expected, Se is located at the outer part and $\mathrm{S}$ is positioned at the inner part confirming the successful growth of CdSe crown layer around the CdS core. In addition, $\mathrm{X}$-ray diffraction spectrum of the CdS core and CdS/CdSe core/ crown NPLs is presented in Figure 2d. XRD diffraction pattern of $4 \mathrm{ML}$ CdS core NPLs exhibits zinc-blend crystal structure with broad diffraction peaks as presumed due to their very thin vertical size. With the formation of CdSe crown region, the diffraction peaks are shifted slightly to the lower angles, which can be attributed to the larger lattice constant of CdSe compared to CdS and formation of strain due to the lattice mismatch between $\mathrm{CdS}$ and CdSe.

The absorption spectra of CdS/CdSe core/crown NPLs at different injection amounts are presented in Figure 3a. The absorption maximum around $410 \mathrm{~nm}$ corresponds to the combined peaks of heavy hole transition (414 nm) and light hole transition $(407 \mathrm{~nm})$ from CdS core region. This absorption peak continues to stay almost at the same wavelength ruling out the possibility of growth in the vertical direction. ${ }^{[25]}$ Lateral growth of the CdSe crown layer on CdS NPLs can be observed in the absorption spectra as a new emerging absorption peak corresponding to the crown layer. Initially, a broad and weak peak associated with the heavy hole and light hole transitions of CdSe crown starts to appear at lower wavelengths, $\approx 465 \mathrm{~nm}$, (a)

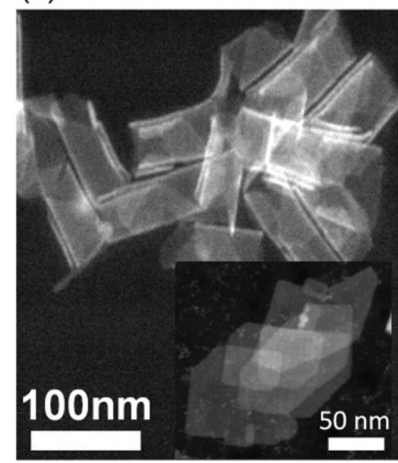

(b)

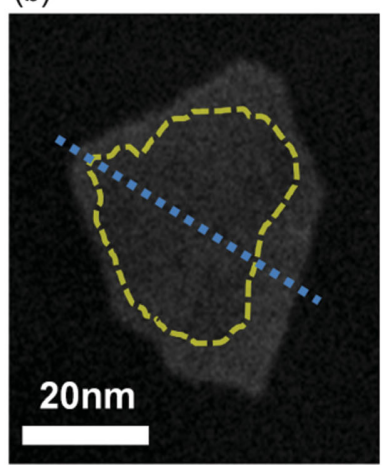

(c)

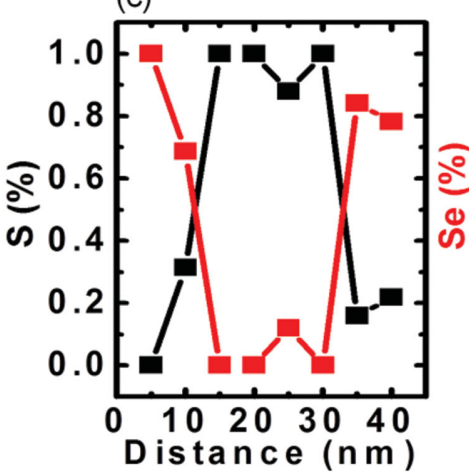

(d)

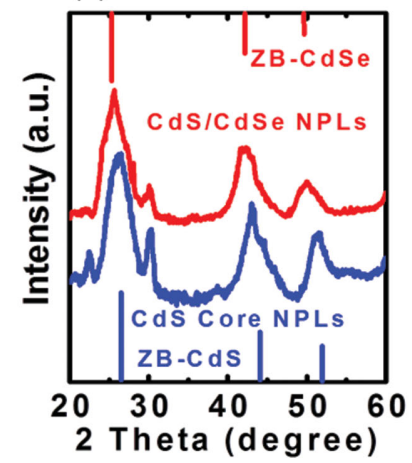

Figure 2. A HAADF-TEM image of a) $4 \mathrm{ML} C d S-C d S e$ NPLs emitting at $515 \mathrm{~nm}$ and b) a $4 \mathrm{ML} \mathrm{CdS} / \mathrm{CdSe}$ NPL with a thin layer of CdSe crown. Yellow dashed line shows the boundary between $\mathrm{CdS}$ and $\mathrm{CdSe}$ layers. c) The distribution of $\mathrm{S}$ and Se concentrations along the blue line attained through line-EDX measurement. d) XRD spectrum of $4 \mathrm{ML} C d S$ (blue) and CdS-CdSe (red) NPLs. 
(a)

(c)
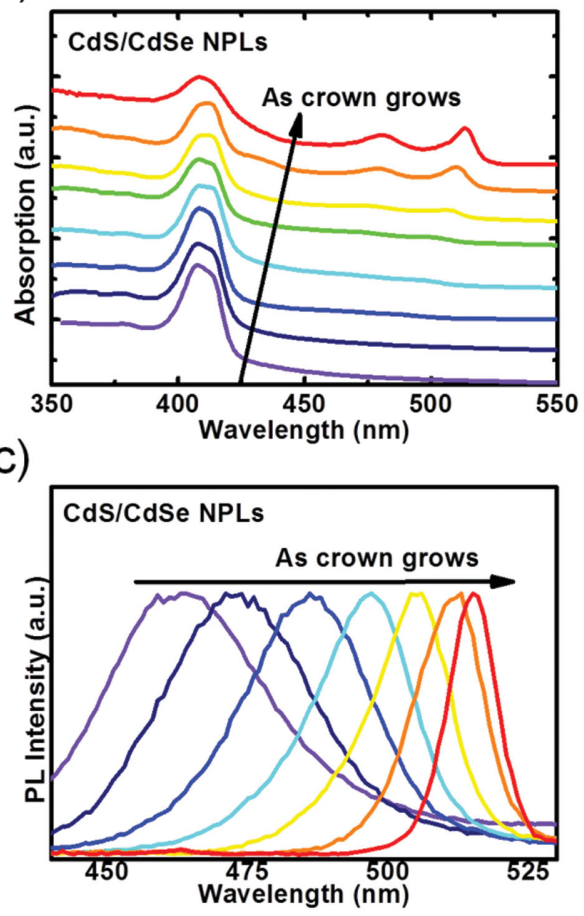

(b)

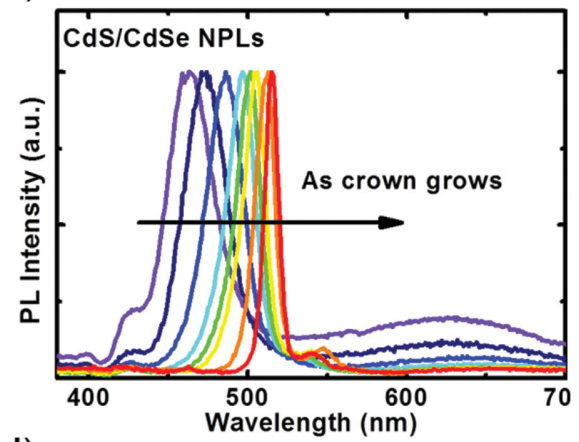

(d)

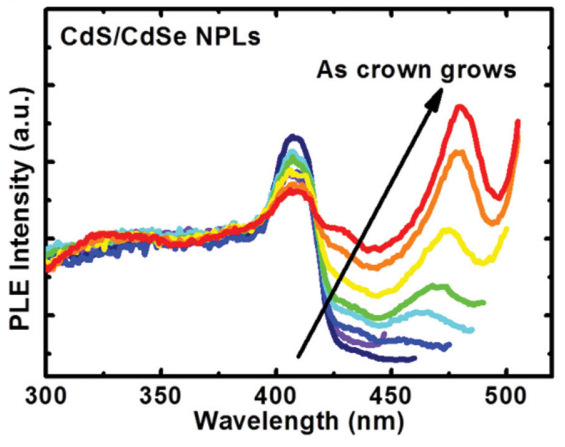

(e)

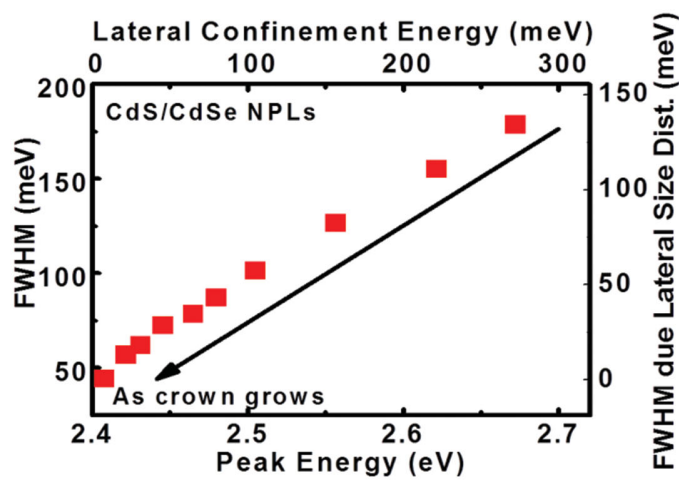

Figure 3. a) Absorption, b) PL, c) PL near the CdSe band edge maxima, and d) PLE at the emission peak maxima measurements of $4 \mathrm{ML} C d / C d S e$ core/crown NPLs. e) FWHM at different emission peaks.

as compared to 4 ML core only CdSe NPLs. We attribute this to the fact that at this initial phase of the injection the size of the crown is small enough so that a strong lateral confinement is present in addition to vertical confinement in the CdSe crown region. The lateral confinement will be discussed in detail in the following sections. With the continuous injection of the crown growth mixture, this new absorption peak corresponding to the excitonic transitions in the CdSe crown starts to slowly red-shift and becomes more pronounced and sharper. This is understood as the crown lateral size is large enough so that the lateral confinement is not strong any more. For the absorption curves corresponding to higher injection amounts, we can identify the heavy hole $(513 \mathrm{~nm})$ and light hole $(481 \mathrm{~nm})$ transitions from the CdSe crown, which are consistent with the peaks appearing in the $4 \mathrm{ML}$ core only CdSe NPLs. The position of the peaks in the absorption spectra confirms that the growth is only in lateral direction. Further, given that a typical synthesis of the core NPLs results in nucleation of more than one species of NPLs which would otherwise appear in the absorption spectrum, the absence of other peaks rules out the possibility of secondary nucleation of new NPLs.

In the CdSe/CdS core/crown NPLs, the energy of the PL emission is almost independent of the size of the CdS crown because of the very strong 1D vertical confinement of the excitons in the CdSe core region. However, since excitons in the CdS/CdSe NPLs are localized at the CdSe crown region, lateral confinement in the crown region can be employed to tailor the optical properties via controlling the lateral size of the CdSe crown. PL emission which is associated with the heavy hole transition in the CdSe crown region in the CdS/CdSe NPLs can be spectrally controlled by varying the crown size. The full PL spectra from the synthesis of the CdS/CdSe core/crown NPLs at various injection amounts are shown in Figure $3 \mathrm{~b}$. The shoulder at $421 \mathrm{~nm}$ in the PL spectra corresponds to the heavy 
hole transition from the CdS cores and diminishes with the continuous injection of CdSe growth mixture. The emission at $421 \mathrm{~nm}$ at the earlier stages of the injection can be attributed to the emission from CdS cores without CdSe crown layer. The emission between 600 and $700 \mathrm{~nm}$ region is attributed to the trap emission in the CdS core NPLs, which is already well known in the literature. This trap emission is eliminated strongly as the crown layer is grown as shown in Figure 3b. This is possibly due to the fact that excitons in the CdS layer are now funneled to the CdSe region within ultrafast time window $(<1 \mathrm{ps}),{ }^{[37]}$ and thus the trap-sites in the CdS cannot be populated. In addition, we present PL spectra focused closely to the band edge emission peak of CdS/CdSe NPLs at various injection amounts as shown in Figure 3c for a better viewing.

Photoluminescence excitation (PLE) measurements at the band edge emission maxima are presented in Figure $3 \mathrm{~d}$ to support that the observed emission comes from the $\mathrm{CdS} / \mathrm{CdSe}$ core/crown NPLs. At the initial phase of the injection, PLE spectra of the peak emission is dominated by the broad peak located at $\approx 408 \mathrm{~nm}$, which is a combination of the heavy hole and light hole transitions of the CdS core at 413 and $405 \mathrm{~nm}$, respectively. Interestingly, for the first two samples having very thin CdSe crown layer, there is no apparent peak in the PLE spectra associated with the excitonic transitions due to $\mathrm{CdSe}$ crown region. This signifies that excitons formed at the $\mathrm{CdS}$ core region are transferred efficiently to the CdSe crown. As we continue to inject the crown growth mixture, the PL emission from the CdS/CdSe NPLs slowly shifts from 460 to $515 \mathrm{~nm}$ and remains constant with the further growth. In addition, in the PLE spectra, peaks associated with the excitonic transitions in the CdSe crown region start to appear and gradually become dominant and sharper as can be seen in Figure 3d. The PL emission at $515 \mathrm{~nm}$ and the light hole transition at $480 \mathrm{~nm}$ is consistent with our observation in the CdSe/CdS core/crown NPLs. Quantum yield of the CdS/CdSe NPLs emitting at $515 \mathrm{~nm}$ was found to be $\approx 55 \%$, which is similar to the core only CdSe NPLs while it is an order of magnitude higher than the core only CdS NPLs. The apparent $2-3 \mathrm{~nm}$ red-shift in the PL and the light hole transition compared to the $4 \mathrm{ML}$ core only CdSe NPLs is due to the relaxation of the wavefunction of excitons in the CdS/CdSe NPLs into the CdS core region owing to a small increase in the dielectric constant.

The FWHM of PL emission in semiconductor nanocrystals strongly depends on the size distribution. NPLs exhibit extremely sharp PL emission compared to colloidal quantum dots owing to their strong 1D confinement and weak lateral confinement. Although the linewidth of CdSe NPLs can be as

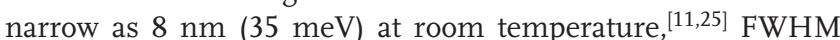
of smaller $4 \mathrm{ML}$ CdS/CdSe NPLs can be as broad as colloidal quantum dots at the initial stage of the crown growth since the lateral confinement is also active. The linewidth of the CdS/ CdSe NPLs as a function of the emission energy is presented in Figure 3e. Linewidth, which is very broad at the initial phase of the injection, becomes sharper with the continuous injection. The linewidth is $28 \mathrm{~nm}$ for CdS/CdSe NPLs emitting at $473 \mathrm{~nm}$ and becomes as narrow as $9.5 \mathrm{~nm}$ for CdS/CdSe NPLs emitting at $515 \mathrm{~nm}$. This emission peak energy dependence of linewidth can be largely attributed to the lateral size distribution of CdSe crown where excitons are localized and confined. Lateral confinement energy is roughly given by the energy difference of PL emission peaks between the NPLs only confined in vertical direction and the NPLs confined as well in lateral direction. Lateral confinement dependence of the linewidth is shown in Figure 3e. Numerical linear fitting to this set of data gives a slope of $\approx 0.51 \pm 0.1$ and intercept of $45 \mathrm{meV}$. Nonzero intercept of $45 \mathrm{meV}$ can be attributed to the intrinsic value of the emission peak in CdS/CdSe core/crown NPLs, which is similar to the observation in the CdSe/CdS core/crown NPLs. ${ }^{[25]}$ FWHM due to the lateral size distribution can be found by subtracting this intrinsic value from the FWHM. As given by the slope, FWHM due to the lateral size distribution is almost half of the confinement energy at all times. For example, at $2.48 \mathrm{eV}$ lateral confinement energy and FWHM due to the lateral size distribution are 80 and $42 \mathrm{meV}$, respectively. As a consequence, the variation in the size of the crown leads to the apparent larger linewidth at smaller crown sizes.

Subsequently, we performed TRF spectroscopy to investigate the fluorescence emission decay kinetics of CdS/CdSe NPLs at room temperature. TRF measurements were performed on NPLs dissolved in hexane under low excitation power $(\langle N\rangle \ll 0.1)$ at room temperature using a pulsed picosecond laser at $3.31 \mathrm{eV}(375 \mathrm{~nm})$. The decay curves were recorded at the peak emission wavelength of each sample. The measured fluorescence decay curves were fitted to a three exponential decay function suggesting multiple decay channels. Results of the fittings (lifetimes, coefficient of each lifetime, average lifetimes and partial contribution of each decay channel) are presented in Table S2, Supporting Information. In a previous work, PL decay curves of single CdSe NPLs were also fitted to the three exponential decay function indicating complex decay kinetics in the CdSe NPLs. ${ }^{[38]}$ Three different lifetime components can be attributed to the radiative channel(s) and nonradiative channel(s), which can be associated with electron or/ and hole trapping. Clear understanding of complex decay kinetics requires more elaborate study on optical properties and as a future work, we intend to carry out a systematic study, including temperature study of QY and PL lifetime components, to investigate the band edge dynamics and exciton trapping in this system. TRF measurements are shown in Figure 4 and amplitude-averaged fluorescence lifetime of the band edge

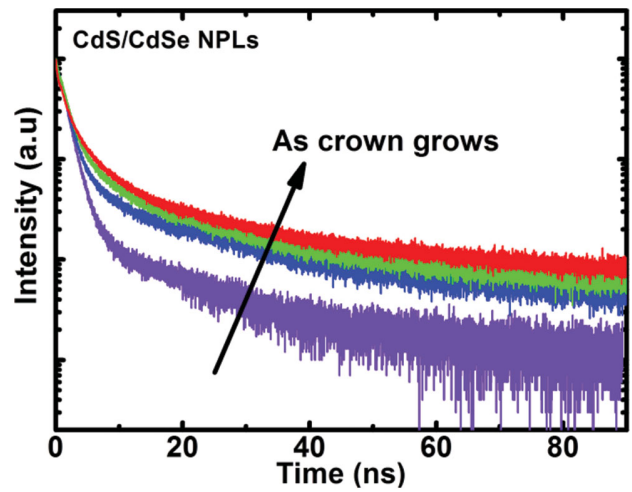

Figure 4. Time-resolved fluorescence decays of the $\mathrm{CdS} / \mathrm{CdSe}$ core/ crown NPLs having different crown sizes at room temperature when the samples are excited at $375 \mathrm{~nm}$. Each decay is collected at the peak emission wavelength of each sample. 
emission increases from 1.6 to $2.1 \mathrm{~ns}$ as the crown layer is further grown larger. The lifetime of $2.1 \mathrm{~ns}$ for the CdS/CdSe NPLs with larger crown sizes is close to the previously reported lifetime of $4 \mathrm{ML}$ CdSe NPLs, $3.5 \mathrm{~ns}$, at room temperature. ${ }^{[11]}$ Changing lateral size of the crown layer would change the oscillatory strength in the CdSe crown region. As the crown size is larger than the exciton Bohr radius of CdSe, the excitons are completely localized in the outer crown layer. Thus, the fluorescence lifetime of these inverted core/crown NPLs become similar to the only core CdSe NPLs with slight difference from the dielectric constant of the core CdS NPLs.

These inverted Type-I NPLs were further investigated for their potential in generating white light emission. The white emission of these NPLs emanates from the narrow-band emission stemming from the CdSe crown of the CdS/CdSe NPLs together with a broadband emission due to the defect states. This concept has been previously studied for quantum dots, ${ }^{[39]}$ however, the potential of the nanoplatelets for white light generation has not been investigated to date. The main differences from the previous works in terms of photophysical features are the sharper blue content in the spectrum and the defect emission from the nanoplatelets as compared to broader blue content from the 3D quantum dots. These differences may have adversely or positively affected the color quality of the emitted light. To study these effects,we synthesized three different 4 ML CdS/CdSe NPL samples having relatively small crowns. The photoluminescence spectra of these NPLs were presented in Figure 5a, along with the corresponding chromaticity coordinates in Figure 5b. The chromaticity points of the emissions belonging to these NPLs show that Samples 1 and 3 acquire a strong bluish emission, which consequently increases their CCT to 8349 and $10794 \mathrm{~K}$, respectively. On the other hand, the stronger yellow-red component of Sample 2 shifts the chromaticity points toward a reddish color point. As a result, CCT of this sample decreases down to $6781 \mathrm{~K}$. The significantly high CCTs of Samples 1 and 3 make them unsuitable for general lighting purposes. However, CCT of Sample 2 remains closer to that of the natural sunlight, making it appropriate for lighting applications. Furthermore, the color rendition performance of the light emitted from these NPLs was evaluated by calculating the color render index (CRI) values. Due to the lack of yellow-red emission, both Samples 1 and 3 acquire very low CRI values (62 and 65, respectively) indicating poor color rendition capability. For Sample 2, however, the strengthened yellowred emission content coming from the broadband emission of NPLs increases the CRI to a reasonably good value of 80 . Therefore, among the NPLs investigated in this particular study, Sample 2 possessing a reasonable CRI with a CCT matching to that of the sunlight can be considered the best for lighting. Considering these results, we can conclude that by controlling the synthesis parameters we can tune the color temperature of NPLs as well as the color.

\section{Conclusions}

In conclusion, we have demonstrated the synthesis and characterization of the first examples of $\mathrm{CdS} / \mathrm{CdSe}$ core/crown NPLs having inverted Type-I structure grown by seed-mediated (a)

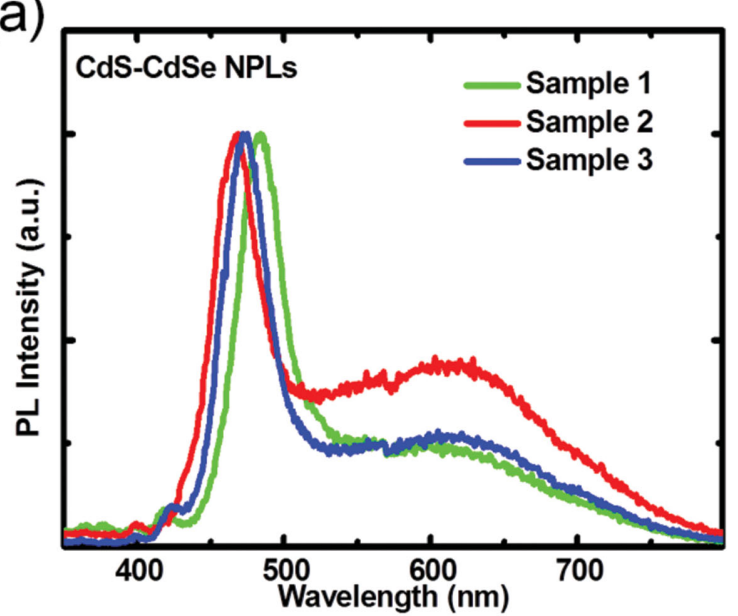

(b)

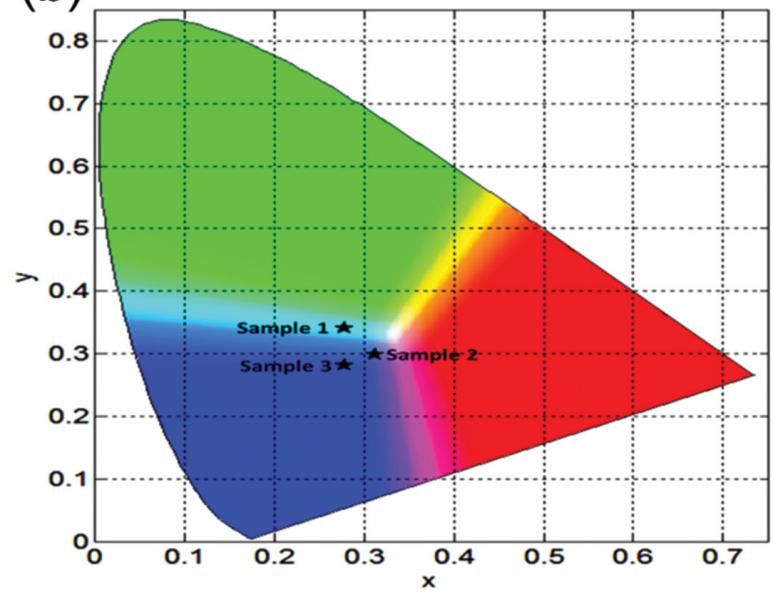

Figure 5. a) Photoluminescence spectra of the white-light-emitting $4 \mathrm{ML}$ $\mathrm{CdS} / \mathrm{CdSe}$ NPLs having different CdSe crown thicknesses. b) The chromaticity coordinates of the white emitting NPLs.

method. We have confirmed that this structure is atomically flat with both the CdS core and CdSe crown regions having the same number of atomic layers. In this heterostructure, excitons collected from the CdS core are funneled efficiently into the CdSe crown owing to the band alignment between CdS and $\mathrm{CdSe}$ and to the large exciton binding energy. In contrast to the CdSe/CdS core/crown NPLs having Type-I band alignment, in the CdS/CdSe core/crown NPLs, the resulting emission peak can be tuned between the emission wavelengths of the core only CdS and CdSe NPLs by controlling the lateral size of the crown. This method can be exploited to realize tunable emission from the NPLs across all visible range by growing the CdSe crown around the CdS NPL cores at various vertical thicknesses. Finally, we demonstrated and evaluated the performance of NPLs with thin crowns for the white light generation. We showed that these NPLs offer reasonable color rendering performance along with reaching a similar CCT to the sunlight. The synthesis of inverted Type-I CdS/CdSe NPLs is significant since it presents a robust method for bandgap engineering in colloidal quantum wells with potential applications in white light generation, photovoltaics, and photoconductivity, and 
initiate a promising approach for the synthesis of colloidal multiple hetero-quantum-wells.

\section{Experimental Section}

Preparation of Cadmium Myristate: Cadmium myristate was synthesized by following the recipe given in the literature. ${ }^{[25]} 1.23 \mathrm{~g}$ of cadmium nitrate tetrahydrate was dissolved in $40 \mathrm{~mL}$ of methanol and $3.13 \mathrm{~g}$ of sodium myristate was dissolved in $250 \mathrm{~mL}$ of methanol under strong stirring. Then, solutions were mixed and stirred around $1 \mathrm{~h}$. The whitish product was centrifuged and the white precipitate part was dissolved in methanol. This washing step with methanol was performed three times for the removal of excess precursors. Subsequently, the final whitish precipitate was kept under vacuum $24 \mathrm{~h}$ for drying.

Synthesis of $4 \mathrm{ML}$ Thick CdS NPLs: $4 \mathrm{ML}$ thick CdS NPLs were synthesized with a slightly modified recipe from the literature. ${ }^{[31]}$ For a typical synthesis, $217 \mathrm{mg}$ of cadmium acetate dihydrate, $2 \mathrm{~mL}$ of $0.1 \mathrm{M}$ of $\mathrm{S}$ in ODE, $0.24 \mathrm{~mL}$ of oleic acid, and $10 \mathrm{~mL}$ of ODE were loaded into a three-neck flask. The solution was stirred under argon flow for $30 \mathrm{~min}$ at room temperature. Under the blanket of argon, the solution was heated to $260{ }^{\circ} \mathrm{C}$ in $20 \mathrm{~min}$ and kept at $260^{\circ} \mathrm{C}$ for $3 \mathrm{~min}$. Then temperature was quickly cooled down to room temperature. CdS NPLs were precipitated with the addition of acetone and dispersed in hexane.

Synthesis of 4 ML Thick CdSe NPLs: 4 ML CdSe NPLs were synthesized following a recipe in the literature. ${ }^{[1]} 170 \mathrm{mg}$ of cadmium myristate, $12 \mathrm{mg}$ of Se, and $15 \mathrm{~mL}$ of ODE were loaded into three-neck flask and degassed for an hour at room temperature. Then, the solution was heated to $240{ }^{\circ} \mathrm{C}$ under the blanket of argon and $80 \mathrm{mg}$ of cadmium acetate dihydrate was injected at $195^{\circ} \mathrm{C}$. The solution was kept at $240^{\circ} \mathrm{C}$ for $10 \mathrm{~min}$ and $0.5 \mathrm{~mL}$ of OA was injected before cooling down to room temperature. CdSe NPLs were precipitated with the addition of acetone and dispersed in hexane.

Preparation of Anisotropic Growth Mixture: Anisotropic growth mixture for $\mathrm{CdSe}$ was prepared with a slightly modified recipe from the literature. ${ }^{[25]} 480 \mathrm{mg}$ of cadmium acetate dihydrate, $340 \mu \mathrm{L}$ of OA, and $2 \mathrm{~mL}$ of ODE were loaded into three-neck flask for the synthesis of cadmium precursor. First, solution was sonicated at room temperature about $30 \mathrm{~min}$. Then, solution was heated to $160{ }^{\circ} \mathrm{C}$ under air with continuous stirring and sonicated frequently during this stage until the formation of homogenous gel having whitish color. $3 \mathrm{~mL} 0.1 \mathrm{~m}$ ODE-Se solution prepared in glovebox was added into cadmium precursor under constant stirring. The final solution was stirred until injection. Similarly, anisotropic growth mixture for CdS was prepared by using $\mathrm{S}$ instead Se.

Synthesis of $4 \mathrm{ML}$ Thick CdS/CdSe Core/Crown NPLs: CdS/CdSe core/ crown NPLs were synthesized via core-seeded method. A portion of $4 \mathrm{ML}$ CdS NPLs synthesis dissolved in hexane and $15 \mathrm{~mL}$ of ODE were loaded into a three-neck flask. The solution was degassed at room temperature for $10 \mathrm{~min}$ and heated up to $80{ }^{\circ} \mathrm{C}$ under vacuum. The solution was kept at $80^{\circ} \mathrm{C}$ about an hour for the complete removal of hexane. Then, the temperature was raised to $255{ }^{\circ} \mathrm{C}$ under the blanket of argon. At $255{ }^{\circ} \mathrm{C}$, anisotropic growth mixture for the extension of $\mathrm{CdSe}$ crown region was injected at a rate of $3 \mathrm{~mL} \mathrm{~h}^{-1}$ mixture. Size of the CdSe crown can be tuned by adjusting the injection amount. After the injection, the temperature was quickly cooled down to room temperature. CdS/ CdSe core/crown NPLs were precipitated with the addition acetone and dispersed in hexane.

Synthesis of 4 ML Thick CdSe/CdS Core/Crown NPLs: CdSe/CdS core/ crown NPLs were synthesized via core-seeded method with a slightly modified recipe from the literature. ${ }^{[25]} \mathrm{A}$ portion of $4 \mathrm{ML}$ CdSe NPLs synthesis dissolved in hexane and $15 \mathrm{~mL}$ of ODE were loaded into a three-neck flask. The solution was degassed at room temperature for $10 \mathrm{~min}$ and heated up to $80^{\circ} \mathrm{C}$ under vacuum. The solution was kept at $80^{\circ} \mathrm{C}$ about an hour for the complete removal of hexane. Then, the temperature was raised to between 230 and $255^{\circ} \mathrm{C}$ under the blanket of argon and anisotropic growth mixture for the extension of CdS crown region was injected at a rate of $3 \mathrm{~mL} \mathrm{~h}$. Size of the $\mathrm{CdS}$ crown can be tuned by adjusting the injection amount. After the injection, the temperature was quickly cooled down to room temperature. CdSe/ CdS core/crown NPLs were precipitated with the addition acetone and dispersed in hexane.

Deposition of One Monolayer CdS Shell on Each Side of NPLs: To unfold NPLs for TEM examination, we benefited from c-ALD technique. ${ }^{[2]}$ NPLs were cleaned before shell deposition as any left-over precursor will react during the first deposition cycle with the reactive $\mathrm{S}^{-2}$ source and cause over-coating of NPLs. After successive cleaning steps, $1 \mathrm{~mL}$ of hexane solution of NPLs and $1 \mathrm{~mL}$ of $\mathrm{N}$-methyl formamide (NFA) were added into a tube. Then, $\mathrm{S}^{-2}$ ions were introduced using aqueous $\left(\mathrm{NH}_{4}\right)_{2} \mathrm{~S}$ precursor. After the addition, color of the NPLs changed spontaneously and phase transfer from hexane to NFA occurred. Then, the NPLs were precipitated out of the solution with the addition of toluene and acetonitrile into the mixture and centrifugation. The supernatant was discarded and the precipitate was dissolved in $1 \mathrm{~mL}$ of NFA. The cleaning step was repeated at least two times to discard the excess precursor molecules. Afterward, NFA solution of cadmium acetate dihydrate was added to the NPL dispersion to deposit $\mathrm{Cd}^{+2}$ ions onto the NPL substrates. The same cleaning procedure was applied to remove the unreacted precursors.

\section{Supporting Information}

Supporting Information is available from the Wiley Online Library or from the author.

\section{Acknowledgements}

The authors would like to thank for the financial support from EU-FP7 Nanophotonics4Energy NoE, and TUBITAK EEEAG 109E002, 109E004, 110E010, 110E217, and 112E183, and from NRF-RF-2009-09, NRF-CRP-6-2010-02, and A*STAR of Singapore. H.V.D. acknowledges support from ESF-EURYI and TUBA-GEBIP.

Received: January 30, 2015

Revised: April 16, 2015

Published online:

[1] S. Coe, W.-K. Woo, M. Bawendi, V. Bulovic, Nature 2002, 420, 800.

[2] V. I. Klimov, A. A. Mikhailovsky, S. Xu, A. Malko, J. A. Hollingsworth, C. A. Leatherdale, H.-J. Eisler, M. G. Bawendi, Science 2000, 290, 314.

[3] W. U. Huynh, J. J. Dittmer, A. P. Alivisatos, Science 2002, 295, 2425.

[4] M. Bruchez, M. Moronne, P. Gin, S. Weiss, A. P. Alivisatos, Science 1998, 281, 2013.

[5] I. Žutić, J. Fabian, S. Das Sarma, Rev. Mod. Phys. 2004, 76, 323.

[6] C. B. Murray, D. J. Norris, M. G. Bawendi, J. Am. Chem. Soc. 1993 $115,8706$.

[7] X. Peng, L. Manna, W. Yang, J. Wickham, E. Scher, A. Kadavanich, A. P. Alivisatos, Nature 2000, 404, 59.

[8] L. Manna, D. J. Milliron, A. Meisel, E. C. Scher, A. P. Alivisatos, Nat. Mater. 2003, 2, 382.

[9] X. Duan, C. M. Lieber, Adv. Mater. 2000, 12, 298.

[10] J. Joo, J. S. Son, S. G. Kwon, J. H. Yu, T. Hyeon, J. Am. Chem. Soc. 2006, 128, 5632.

[11] S. Ithurria, M. D. Tessier, B. Mahler, R. P. S. M. Lobo, B. Dubertret, A. L. Efros, Nat. Mater. 2011, 10, 936.

[12] B. Guzelturk, P. L. H. Martinez, Q. Zhang, Q. Xiong, H. Sun, X. W. Sun, A. O. Govorov, H. V. Demir, Laser Photonics Rev. 2014 8,73 
[13] P. Reiss, J. Bleuse, A. Pron, Nano Lett. 2002, 2, 781.

[14] L. P. Balet, S. A. Ivanov, A. Piryatinski, M. Achermann, V. I. Klimov, Nano Lett. 2004, 4, 1485.

[15] M. A. Hines, P. Guyot-Sionnest, J. Phys. Chem. 1996, 100, 468.

[16] X. Peng, M. C. Schlamp, A. V. Kadavanich, A. P. Alivisatos, J. Am. Chem. Soc. 1997, 119, 7019.

[17] B. Mahler, P. Spinicelli, S. Buil, X. Quelin, J.-P. Hermier, B. Dubertret, Nat. Mater. 2008, 7, 659.

[18] O. Chen, J. Zhao, V. P. Chauhan, J. Cui, C. Wong, D. K. Harris, H. Wei, H.-S. Han, D. Fukumura, R. K. Jain, M. G. Bawendi, Nat. Mater. 2013, 12, 445.

[19] M. C. Schlamp, X. Peng, A. P. Alivisatos, J. Appl. Phys. 1997, 82, 5837.

[20] a) S. A. Ivanov, J. Nanda, A. Piryatinski, M. Achermann, L. P. Balet, I. V. Bezel, P. O. Anikeeva, S. Tretiak, V. I. Klimov, J. Phys. Chem. B 2004, 108, 10625; b) Y. Kelestemur, A. F. Cihan, B. Guzelturk, H. V. Demir, Nanoscale 2014, 6, 8509.

[21] a) Y.-L. Lee, Y.-S. Lo, Adv. Funct. Mater. 2009, 19, 604; b) Z. Pan, H. Zhang, K. Cheng, Y. Hou, J. Hua, X. Zhong, ACS Nano 2012, 6, 3982.

[22] a) M. A. Hossain, J. R. Jennings, Z. Y. Koh, Q. Wang, ACS Nano 2011, 5, 3172; b) X.-Y. Yu, J.-Y. Liao, K.-Q. Qiu, D.-B. Kuang, C.-Y. Su, ACS Nano 2011, 5, 9494.

[23] a) A. Mews, A. Eychmueller, M. Giersig, D. Schooss, H. Weller, J. Phys. Chem. 1994, 98, 934; b) M. Braun, C. Burda, M. A. El-Sayed, J. Phys. Chem. A 2001, 105, 5548.

[24] D. Battaglia, J. J. Li, Y. Wang, X. Peng, Angew. Chem. Int. Ed. 2003, 42, 5035.
[25] M. D. Tessier, P. Spinicelli, D. Dupont, G. Patriarche, S. Ithurria, B. Dubertret, Nano Lett. 2013, 14, 207.

[26] B. Mahler, B. Nadal, C. Bouet, G. Patriarche, B. Dubertret, J. Am. Chem. Soc. 2012, 134, 18591.

[27] S. Ithurria, D. V. Talapin, J. Am. Chem. Soc. 2012, 134, 18585

[28] S. Ithurria, B. Dubertret, J. Am. Chem. Soc. 2008, 130, 16504.

[29] M. D. Tessier, B. Mahler, B. Nadal, H. Heuclin, S. Pedetti, B. Dubertret, Nano Lett. 2013, 13, 3321.

[30] a) B. Guzelturk, Y. Kelestemur, M. Olutas, S. Delikanli, H. V. Demir, ACS Nano 2014, 8, 6599; b) A. Prudnikau, A. Chuvilin, M. Artemyev, J. Am. Chem. Soc. 2013, 135, 14476.

[31] J. C. de Mello, H. F. Wittmann, R. H. Friend, Adv. Mater. 1997, 9 , 230.

[32] Z. Li, H. Qin, D. Guzun, M. Benamara, G. Salamo, X. Peng, Nano Res. 2012, 5, 337.

[33] C. Bouet, B. Mahler, B. Nadal, B. Abecassis, M. D. Tessier, S. Ithurria, X. Xu, B. Dubertret, Chem. Mater. 2013, 25, 639.

[34] A. Pandey, P. Guyot-Sionnest, J. Chem. Phys. 2007, 127, 104710.

[35] C. Javaux, B. Mahler, B. Dubertret, A. Shabaev, A. V. Rodina, A. L. Efros, D. R. Yakovlev, F. Liu, M. Bayer, G. Camps, L. Biadala, S. Buil, X. Quelin, J. P. Hermier, Nat. Nanotechnol. 2013, 8, 206.

[36] G. Rainò, T. Stöferle, I. Moreels, R. Gomes, J. S. Kamal, Z. Hens, R. F. Mahrt, ACS Nano 2011, 5, 4031.

[37] M. G. Lupo, F. Della Sala, L. Carbone, M. Zavelani-Rossi, A. Fiore, L. Lüer, D. Polli, R. Cingolani, L. Manna, G. Lanzani, Nano Lett. 2008, 8, 4582

[38] M. D. Tessier, C. Javaux, I. Maksimovic, V. Loriette, B. Dubertret, ACS Nano 2012, 6, 6751 .

[39] T. Erdem, H. V. Demir, Nanophotonics 2013, 2, 57. 